\title{
PRODUÇÃO CIENTÍFICA E TECNOLÓGICA DAS REGIÕES METROPOLITANAS BRASILEIRAS $*$
}

\author{
Eduardo da Motta e Albuquerque \\ Adriano Ricardo Baessa ${ }^{* *}$ \\ João Carlos Vieira Kirdeikas ${ }^{* * * *}$ \\ Leandro Alves Silva \\ Ricardo Machado Ruiz
}

\begin{abstract}
RESUMO Este artigo faz uma avaliação da produção científica e tecnológica das regiões metropolitanas no Brasil a partir de estatísticas de patentes (depositadas no INPI) e de artigos científicos (indexados pelo ISI). O trabalho está dividido em cinco seções. A seção 1 resenha brevemente a literatura sobre interação entre ciência e tecnologia, desde o ponto de vista da abordagem dos sistemas nacionais de inovação. A seção 2 apresenta as bases de dados e um quadro geral da produção científica e tecnológica do Brasil. A seção 3 focaliza as regiões metropolitanas do Brasil, avaliando a distribuição das atividades científicas e tecnológicas entre elas e comparando-a com outros países. A seção 4 avalia as diferentes especializações científicas e tecnológicas das regiões metropolitanas avaliadas. A seção 5 conclui o trabalho.
\end{abstract}

Palavras-chave: estatísticas de ciência e tecnologia; sistema nacional de inovação; distribuição espacial

Código JEL: O0, R00

* Artigo recebido em 27 de janeiro de 2005 e aprovado em $1^{\circ}$ de setembro de 2005 . Este texto foi produzido a partir de pesquisa contratada pelo Parque Tecnológico da UFMG (com recursos da Finep e da Fapemig). Os autores agradecem o apoio dos bolsistas de iniciação científica Cintia Oliveira, Hérica Righi, Camila Lins, Ana Luíza Lara, Elaine Rodrigues, Raquel Guimarães, Thaís Henriques e Ricardo Carvalho. Comentários teóricos e sugestões empíricas de Fabiana Santos (Parque Tecnológico da UFMG), Clélio Campolina Diniz e Mauro Borges Lemos (Cedeplar/ UFMG) a uma versão preliminar deste artigo foram de grande estima. Os autores agradecem as críticas e sugestões de dois pareceristas anônimos da Revista de Economia Contemporânea. Os erros são de responsabilidade exclusiva dos autores.

** Eduardo da Motta e Albuquerque (Cedeplar/UFMG), e-mail: albuquer@cedeplar.ufmg.br

*** Adriano Ricardo Baessa (IPEA), e-mail: adriano.baessa@ipea.gov.br

$\star * * *$ João Carlos Vieira Kirdeikas (Cedeplar/UFMG), e-mail: kirk@cedeplar.ufmg.br

$* * * * *$ Leandro Alves Silva (Cedeplar/UFMG), e-mail: 1silva@cedeplar.ufmg.br

$\star \star \star \star * *$ Ricardo Machado Ruiz (Cedeplar/UFMG), e-mail: rmruiz@cedeplar.ufmg.br 


\section{THE SCIENTIFIC AND TECHNOLOGICAL PRODUCTION OF BRAZILIAN METROPOLITAN}

ABSTRACT This paper evaluates the scientific and technological production of Metropolitan Areas in Brazil. The data are from the Brazilian Patent Office (Instituto Nacional de Propriedade Industrial - INPI) and from the Institute for Scientific Information (ISI). The first section surveys the literature on interactions between universities and firms. The second section presents the data bases and a general picture of the Brazilian scientific and technological production. The third section focuses the Metropolitan Areas. The fourth section evaluates the different scientific and technological specializations of these Areas. The fifth section concludes the paper, discussion connections and disconnections in the Brazilian National System of Innovation.

Key words: science and technology statistics; national system of innovation; spatial distribution 


\section{INTRODUÇÃO}

A co-localização geográfica da produção científica e tecnológica brasileira é apresentada em nível metropolitano. Zitt et al. (2003) apresentam estudo sobre a co-localização geográfica da produção científica e tecnológica na União Européia como uma forma de investigar o potencial para spill-overs. Este texto investiga a co-localização geográfica em nível metropolitano como uma forma de avaliar a existência de conexão (ou desconexão) entre a produção científica e a produção tecnológica.

O Brasil produz 1,4\% da ciência mundial e $0,1 \%$ da tecnologia mundial, considerando as participações relativas da produção nacional no total mundial de artigos científicos indexados no ISI e de patentes depositadas no USPTO em 2001. Essa comparação elementar entre dados relativos à dimensão científica e tecnológica sugere que o Brasil pode estar desperdiçando oportunidades tecnológicas geradas pela presente acumulação científica nacional. O setor produtivo parece não utilizar de forma intensiva os conhecimentos disponibilizados pela infra-estrutura científica existente no país.

A disparidade entre a participação relativa do Brasil na produção científica mundial e sua participação na produção tecnológica mundial, característica compartilhada com outros países em posição tecnológica intermediária como a Índia, a África do Sul e o México, não pode alimentar ilusões: é ainda pequena a participação nacional na produção científica mundial. Para identificar essa posição, duas comparações podem ser realizadas: em primeiro lugar, o PIB do Brasil representou 2,53\% do Produto Mundial Bruto em 1999; em segundo lugar, o Brasil produziu 63 artigos por milhão de habitantes em 1998, enquanto o limiar para uma interação positiva entre ciência e tecnologia encontrava-se em 163 artigos por milhão de habitantes (Bernardes e Albuquerque, 2003).

Ao mesmo tempo em que possível, dada a disparidade apontada acima, o maior envolvimento do setor produtivo com atividades inovativas é importante para o crescimento da própria atividade científica, pois o setor produtivo é uma importante fonte de questões, problemas e perguntas que alimentam a infra-estrutura científica em sistemas de inovação mais completos e articulados. E vale notar que um maior envolvimento do setor produtivo em atividades inovativas é, por certo, um requisito de qualquer política industrial voltada a ganhos sustentados de competitividade. 


\section{SISTEMAS DE INOVAÇÃO E A INTERAÇÃO ENTRE CIÊNCIA E TECNOLOGIA'}

Um sistema nacional de inovação é uma construção institucional, produto seja de uma ação planejada e consciente, seja de um somatório de decisões descentalizadas e desarticuladas, que impulsiona o progresso tecnológico em economias capitalistas complexas (Freeman, 1987; Nelson, 1993). Pelo sistema de inovação viabilizam-se os fluxos de informação e infra-estrutura necessários ao processo de inovação tecnológica. Estes arranjos institucionais envolvem firmas, redes de interação entre empresas, agências governamentais, universidades, institutos de pesquisa, laboratórios de empresas, atividades de cientistas e engenheiros.

As universidades, instituições de pesquisa e o conjunto da estrutura educacional são cruciais para a manutenção do um sistema de inovação. A National Science Foundation (NSF, 1996) estima que nos Estados Unidos o total de cientistas e engenheiros empregados em atividades de P\&D alcançou a cifra de 962.700 pessoas em 1993 (cerca de 1\% da força de trabalho do país). O total de cientistas e engenheiros empregados em atividades não acadêmicas atingiu 3.502.000 (1.749.000 cientistas e 1.753 .000 engenheiros), cerca de 3\% da força de trabalho em 1992.

Essas estruturas de pesquisa articulam-se com o sistema educacional, com o setor industrial e empresarial e, também, com as instituições financeiras, completando o circuito dos agentes que são responsáveis pela geração, implementação e difusão das inovações. Um sistema de inovação diversifica a divisão tecnológica de trabalho, fornecendo às firmas oportunidades tecnológicas de forma persistente, por isso são considerados por muitos estudiosos um ponto focal de qualquer política de desenvolvimento econômico. ${ }^{2}$

A estrutura dos sistemas de inovação difere entre os países (Nelson, 1993). Estudos comparativos permitem distinguir a existência de pelo menos quatro grupos de países com padrões distintos de maturidade de seus sistemas de inovação.

Em primeiro lugar, o grupo dos países avançados, no qual os sistemas de inovação completaram o seu processo de construção, constituindo-se como sistemas maduros. Neles, a articulação institucional entre o sistema científico-tecnológico, o sistema financeiro e o sistema educacional alcançou um 
grau tal que torna o progresso tecnológico a principal fonte de desenvolvimento econômico. Os Estados Unidos seriam um perfeito representante desse grupo.

Em segundo lugar, há um grupo de países em processo de catching up, como a Coréia do Sul e Taiwan que, nas últimas três décadas, foram um exemplo da importância de instituições de apoio às atividades científicas e tecnológicas (Amsden, 1989; Wade, 1990). A avaliação dos processos de desenvolvimento desses países indica que a construção e o amadurecimento de sistemas de inovação constituem pré-requisitos para um desenvolvimento econômico sustentado.

O terceiro grupo reúne países heterogêneos, como o Brasil, a Índia, o México, a África do Sul, a Rússia e a Malásia. Esses países possuem um sistema de inovação com a presença de elementos de infra-estrutura científica e alguma capacidade tecnológica do setor produtivo.

No quarto grupo encontram-se as nações mais pobres do mundo, segundo a classificação do Banco Mundial, que na melhor das hipóteses possuiriam sistemas de inovação rudimentares (Banze, 2000). ${ }^{3}$

\subsection{Limiares e interações entre a ciência e a tecnologia}

Para os objetivos deste trabalho, é conveniente discutir com mais detalhes o papel da infra-estrutura científica para o processo de desenvolvimento econômico, aprofundando a compreensão das interações entre a dimensão científica (a esfera das universidades, dos institutos de pesquisa) e a dimensão tecnológica (a esfera das firmas, de seus laboratórios de P\&D).

Apesar de serem distintas, estas duas esferas participam de uma complexa divisão de trabalho que responde pela sustentação do dinamismo dos sistemas de inovação. Importa, sobretudo, compreender o papel da interação entre elas.

\subsubsection{Ciência e tecnologia nos sistemas de inovação maduros}

Nelson e Rosenberg (1993) apontam o entrelaçamento entre as dimensões científica e tecnológica como uma das características principais dos sistemas de inovação. Resumem as complexas interações entre essas duas dimensões ao afirmar que a ciência tanto "lidera como segue" (science as a leader and a follower) o progresso tecnológico (p. 6). 
Rosenberg (1982: 141-159) discute "quão exógena é a ciência": ressalta como os fatores econômicos determinam, até certo ponto, o progresso da ciência, e que (1) a tecnologia é uma fonte de questões e problemas para a ciência; (2) a tecnologia é um "enorme depósito de conhecimento empírico para ser investigado e avaliado pelos cientistas" (p. 144); (3) o progresso tecnológico contribui para a definição de uma agenda de pesquisa científica; (4) avanços técnicos disponibilizam novos instrumentos e equipamentos para atividade científica. Rosenberg conclui que existem "poderosos impulsos econômicos moldando, dirigindo e constrangendo o empreendimento científico" (p. 159).

Em segundo lugar, Klevorick et al. (1995) apresentam evidências empíricas do papel das universidades e da ciência como fontes de oportunidades tecnológicas para a inovação industrial. O estudo mostra como os diferentes setores industriais avaliam a importância relativa das universidades e da ciência para a sua capacitação inovativa e permite compreender por que as firmas gastam recursos próprios para monitorar e acompanhar a evolução da pesquisa acadêmica. Especialmente em áreas de alta tecnologia, identificam-se intensos fluxos de conhecimento fluindo da infra-estrutura científica para os setores industriais.

Em terceiro lugar, para Pavitt (1991: 114), além da sua utilidade como um "insumo de importância crescente para a tecnologia", a pesquisa básica tem duas outras influências talvez até mais significativas: (1) contribui para o desenvolvimento de habilidades de pesquisa e treina pesquisadores que posteriormente são empregados no setor industrial; (2) é fonte de aplicações não planejadas.

Em quarto lugar, Rosenberg (1990) discute "por que firmas fazem pesquisa básica" e sugere que ela é "um bilhete de entrada para uma rede de informações". Esse ponto está relacionado à discussão de Cohen e Levinthal (1989) sobre as duas faces do processo de P\&D, destacando a importância desse investimento para o desenvolvimento de "capacidade de absorção". O investimento em P\&D é, simultaneamente, investimento para inovação e para aprendizado.

Em quinto lugar, Narin et al. (1997) encontram evidências empíricas do vínculo crescente entre a ciência (fundamentalmente financiada pelo setor público) e a capacidade inovativa da indústria dos Estados Unidos. 
Em resumo, estes estudos demonstram a relevância das duas dimensões da atividade inovativa, enfatizando a divisão de trabalho entre elas e identificando a presença de uma forte interação e de retroalimentação (feedbacks) entre a infra-estrutura científica e a produção tecnológica nos países desenvolvidos. Importa ainda frisar que esta literatura sugere que para assegurar a sustentabilidade do crescimento econômico moderno, estas interações devem estar operando de forma intensa. ${ }^{4}$

\subsubsection{O papel da ciência no processo de catching up}

Por outro lado, a situação prevalecente nos países menos desenvolvidos não pode ser compreendida a partir da aplicação direta e sem qualificações das conclusões alcançadas na literatura sobre os países avançados. Há diferenças que devem ser consideradas e ponderadas.

No que diz respeito ao papel da ciência, a principal diferença reside na contribuição que ela pode oferecer ao processo de catching up: a infra-estrutura científica atua como um "instrumento de focalização" e como uma "antena” para identificar oportunidades tecnológicas e para constituir a capacidade de absorção do país. Em um país atrasado, a infra-estrutura científica oferece "conhecimento para focalizar buscas" (Nelson, 1982), em vez de ser uma fonte direta de oportunidades tecnológicas. Em outras palavras, a infra-estrutura científica em países em desenvolvimento deve contribuir para vincular o país aos fluxos científicos e tecnológicos internacionais.

Neste sentido, o papel da ciência durante processos de catching up pode ser desdobrado em três dimensões. Em primeiro lugar, ela atua como um "instrumento de focalização" contribuindo para a identificação de oportunidades e para a vinculação do país aos fluxos internacionais. Em segundo lugar, a ciência cumpre o papel de instrumento de apoio para o desenvolvimento industrial, provendo conhecimento necessário para a entrada em setores industriais estratégicos (Perez e Soete, 1988). Finalmente, ela serve como fonte para algumas soluções criativas que dificilmente seriam obtidas fora do país. Certamente há uma inter-relação entre esses diferentes papéis, na medida em que o desenvolvimento da capacidade de absorção é uma precondição para desenvolvimentos tecnológicos locais, incrementais e originais.

Comparando as interações entre as esferas da ciência e da tecnologia em países em processo de catching up (Coréia do Sul e Taiwan) com o caso bra- 
sileiro, Rapini (2000) encontrou uma importante diferença: nos países em catching up é possível estabelecer uma relação estatística que sugere a existência de causalidade tanto entre a produção científica (em termos de artigos científicos indexados pelo ISI) e a produção tecnológica (em termos de patentes depositadas no USPTO) como entre a produção tecnológica e a produção científica. ${ }^{5}$ Ou seja, identificou-se uma causalidade recíproca entre as dimensões científica e tecnológica ao longo do processo de desenvolvimento acelerado. Esse achado contrasta com o caso brasileiro, no qual a autora encontrou causalidade em apenas um sentido: da produção científica para a tecnológica.

\subsubsection{Os limiares de produção científica}

A constatação desta diferença entre a dinâmica das esferas científica e tecnológica nos países em catching up e no Brasil aponta a necessidade de investigar as condições necessárias para que se estabeleça uma interação, uma relação recíproca entre estas esferas tal como aquela que caracteriza os sistemas de inovação maduros.

Neste sentido, assume-se aqui a proposição de Bernardes e Albuquerque (2003) sobre a existência de pelo menos três "regimes de interação". No Regime I, a infra-estrutura científica é ainda muito pequena e incapaz de alimentar uma produção tecnológica mínima. ${ }^{6}$ No Regime II, a produção científica cresce e pode determinar alguma produção tecnológica, mas não a ponto de viabilizar um efeito retroalimentador sobre a produção científica. ${ }^{7}$ Finalmente, no Regime III, as conexões e interações estão plenamente estabelecidas e o principal determinante do crescimento econômico é a capacitação científica e tecnológica.

Desta forma, Bernardes e Albuquerque (2003) defendem a necessidade dos países em catching up de alcançarem uma certa "massa crítica" na produção científica, a fim de passar para um estágio superior de interação entre a dimensão científica e a tecnológica. Os achados de Bernardes e Albuquerque (2003) podem ser resumidos no gráfico 1, que apresenta dados referentes à produção científica (artigos por milhão de habitantes, $A^{*}$ ) e à produção tecnológica (patentes por milhão de habitantes, $\mathrm{P}^{\star}$ ) de 120 países que produziram ao menos uma patente e um artigo em 1998. Ele sugere a existência de um ponto a partir do qual a "eficiência" na transformação de arti- 


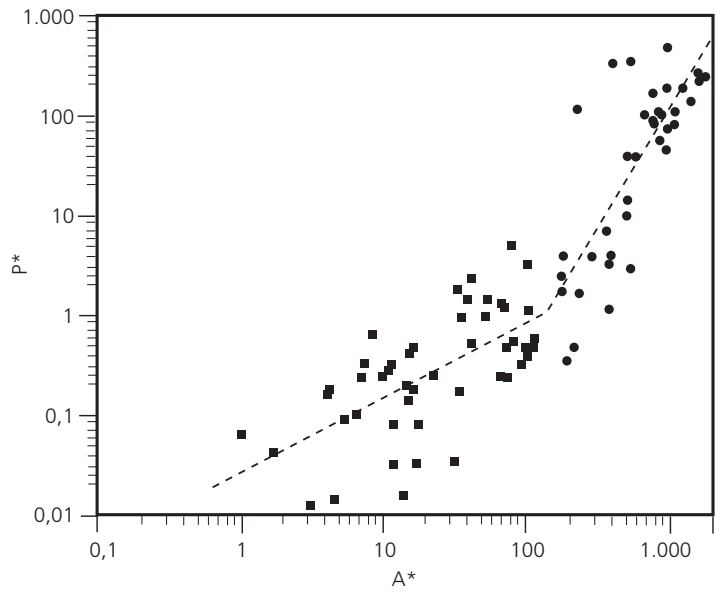

Notas: $A^{*}$ : Log-log de artigos por milhão de habitantes. $P^{*}$ : Patentes por milhão de habitantes. Fonte: Bernardes e Albuquerque (2003)

gos em patentes sofre uma ruptura. A vizinhança da produção de 150 artigos por milhão de habitantes parece constituir-se no limiar de produção científica, para os dados de 1998.

A investigação do comportamento desta relação para outros anos (1974, 1982 e 1990) também indica um padrão similar, mas sugere que o limiar tem se movimentado ao longo do tempo: em 1974, 7 artigos por milhão de habitantes; em 1982, 28; e, em 1990, 60. Essa movimentação do limiar de produção tecnológica pode ser interpretada como um aumento das exigências absolutas em termos de infra-estrutura científica para processos de catching up. ${ }^{8}$

\subsection{Proximidade geográfica e difusão tecnológica}

Conforme vimos, a interação entre ciência e tecnologia é decisiva. Ela envolve instituições constitutivas dos sistemas de inovação - universidades e instituições de pesquisa, por um lado, e firmas, por outro - e tem uma dimensão espacial, donde é importante uma avaliação sumária do papel da proximidade geográfica para a implementação dos fluxos tecnológicos interinstitucionais. Aliás, o estudo de Cohen et al. (2002: 15) já indica o papel da "interação informal”, que depende da proximidade geográfica. 
Uma conexão inicial entre o papel das cidades e as atividades inovativas pode ser estabelecida a partir do papel da proximidade geográfica para a articulação entre esses dois componentes do sistema de inovação. Tema também discutido pela literatura sobre sistemas locais/regionais de inovação e pela literatura sobre os spillovers espaciais de conhecimento.

Uma articulação entre a literatura de sistemas de inovação e o papel da proximidade geográfica é oferecida pela elaboração de Johnson e Lundvall (2005). Discutindo o papel do aprendizado na construção de uma agenda nova e mais complexa para responder aos desafios da globalização, esses autores destacam o papel da esfera regional para ampliação da capacidade de absorção de mudanças. Nessa nova agenda, enfatizam: "Devem ser priorizadas as políticas que visam ao desenvolvimento de recursos humanos, as novas formas de organização, a implementação de redes inovadoras, a reorientação das políticas de inovação na direção dos setores de serviços e a integração de universidades aos processos de inovação" (p. 124).

Audretsch e Feldman (1996: 639) recolhem evidências que indicam que “(...) indústrias nas quais os spillovers de conhecimento são mais prevalentes - isto é, cujo P\&D industrial, a pesquisa universitária e o trabalho especializado são mais importantes - têm uma maior propensão de concentrar espacialmente as atividades inovativas do que indústrias onde as externalidades de conhecimento são menos importantes". Esse argumento é, em grande medida, corroborado por Jaffe (1989), que discute a importância da proximidade geográfica entre universidades e firmas para a economia dos Estados Unidos.

Ainda do ponto de vista de uma ênfase na proximidade como fator de difusão científica e tecnológica, Jaffe et al. (1993) analisam a localização de patentes e citações como evidência de spillovers espaciais. Mostram que a dinâmica de citações de patentes e referências segue um padrão mais vinculado à proximidade do que à concentração relativa de atividades correlatas de pesquisa. Paralelamente, Wallsten (2001) explora as relações de aglomeração e spillovers geográficos da indústria do conhecimento, concluindo também que a proximidade influencia fortemente a difusão das atividades de P\&D.

De um ponto de vista eminentemente espacial, vários autores relacionam a vinculação das atividades em $P \& D$ com a presença de equipamentos e 
serviços urbanos, além da própria densidade econômica do espaço como fatores de atração. Camagni (1985) utiliza argumentos marshalianos e discute a dimensão aglomerativa relacionada às atividades de $\mathrm{P} \& \mathrm{D}$ e sua influência no crescimento das empresas. Malecki (1984), Markussen et al. (1986) e Angel (1989) relacionam a densidade econômica urbana com a atração de cientistas e tecnólogos para determinadas áreas. Por sua vez, Sivitanidou e Sivitanides (1995) utilizam variáveis relacionadas às chamadas amenidades urbanas — segundo Gottlieb (1995: 1.413) “(...) amenities may be defined as place-specific goods or services that enter the utility functions of residents directly" - para o estudo da distribuição espacial intrametropolitana de atividades de P\&D para os Estados Unidos. Frenkel (2001) avalia a localização de firmas de alta tecnologia concluindo que o milieu productive exerce influência destacada nesta localização, enfatizando tanto o papel da infra-estrutura como o dos serviços financeiros e da própria densidade urbana relacionada à aglomeração econômica regional.

Como ilustrado acima, existe uma vasta literatura sobre as aglomerações espaciais e atividades de inovação. Em geral, os argumentos classificam essa interação espacial como uma força centrípeta ou polarizadora, uma externalidade positiva no-tradable e uma fonte para os clássicos rendimentos crescentes de escala Kaldorianos e os ciclos virtuosos de Verdoom (Feldman e Audrestch, 1999, p. 410). Em outras palavaras, a troca de conhecimentos entre indivíduos, instituições e firmas que compartilham o mesmo espaço econômico amplifica e gera rendimentos crescentes de escala. Sendo assim, os sistemas nacionais de inovação possuem uma dimensão espacial que sinaliza para uma polarização geográfica das instituições que os compõem (Carlino et al., 2001).

\section{BASES DE DADOS E METODOLOGIA}

As bases de dados utilizadas envolvem patentes (para avaliar a dimensão tecnológica) e artigos científicos (para avaliar a dimensão científica). A espacialização desses dados possibilitará uma indicação preliminar das interações espaciais entre universidades/instituições de pesquisa e empresas. A espacialização dos dados permite investigar a co-localização geográfica da 
produção científica e tecnológica (Zitt et al., 2003). Esse trabalho permitirá detalhar algumas informações úteis para uma melhor "fotografia" do estágio atual do sistema de inovação brasileiro.

Como proxies de produção científica utiliza-se, geralmente, o número de artigos científicos publicados, o número de pesquisadores etc. E para a produção tecnológica utilizam-se como proxy patentes depositadas ou concedidas pelos escritórios de propriedade industrial ou intelectual. No presente trabalho, os dados de artigos científicos foram retirados do Institute for Scientific Information (ISI, www.isiknowledge.com). As informações de patentes depositadas entre 1990 e 2000 foram cedidas pelo Instituto Nacional de Propriedade Industrial (INPI). Essa base envolve um total de 49.138 patentes, das quais 37.772 foram depositadas por pessoas físicas e 11.366 por pessoas jurídicas. Além do titular, as informações cedidas pelo INPI indicam a classe tecnológica da patente, de acordo com a Classificação Internacional de Patentes, definida pela Organização Mundial de Propriedade Intelectual (OMPI). Aqui optou-se por trabalhar com a classe tecnológica. O INPI informou apenas a unidade da federação do titular, donde foi necessário um processamento especial para a identificação dos titulares, que foi realizado a partir do cruzamento da razão social destes na RAIS (Relação Anual de Informação Social) de 1997.

Distorções, portanto, são implícitas neste processo: (1) das 5.281 firmas/ instituições que detinham as 11.366 patentes de pessoas jurídicas, foram identificadas junto ao banco da RAIS 2.882 firmas e instituições que depositaram 8.153 patentes; (2) quando uma mesma firma, com unidades em municípios numa mesma UF, era encontrada, seu município tinha de ser "arbitrado" (o critério utilizado é a escolha da unidade com maior número de empregados). Quanto aos dados de pesquisadores, estes foram obtidos no CNPq, mais precisamente na configuração dos grupos de pesquisa em $2000 .{ }^{9}$

\section{REGIÕES METROPOLITANAS E ATIVIDADES DE C\&T}

Nesta seção, a investigação concentra-se na comparação das participações relativas das regiões metropolitanas. Investigação que é uma contribuição para a avaliação do grau de concentração das atividades de ciência e tecno- 
logia no sistema de inovação brasileiro. Para tanto, inicialmente uma comparação internacional é apresentada, para que a pergunta acerca do grau de descentralização das atividades de C\&T no país possa ser respondida de forma comparativa. Posteriormente, a participação relativa das regiões metropolitanas e microrregiões é detalhada.

Um dos aspectos a ressaltar na comparação internacional é o padrão de distribuição regional das atividades de P\&D entre países.

BMBF (1999: 89) apresenta a porcentagem do pessoal empregado em atividades de $\mathrm{P} \& \mathrm{D}$ nas principais regiões metropolitanas de países avançados. No texto, são identificados três padrões diferentes de distribuição de atividades inovativas (BMBF, 1999: 89-90). Em primeiro lugar, há a "concentração monocêntrica": é o caso da maior parte dos países europeus, envolvendo países pequenos (exemplo, Finlândia, onde a região de Helsinki concentra 47\% do pessoal de P\&D) e países maiores (como Grã-Bretanha e a França, onde Londres e Paris concentram mais de 41\% do pessoal de P\&D).

Em segundo lugar, há a "concentração oligocêntrica", exemplificada pelos casos da Itália (Lombardia com 32\%, Piemonte com 24\% e região de Lazio com 10\% do pessoal empregado) e da Espanha (Madri com 32\% e Barcelona com 29\%).

Em terceiro lugar, há a "concentração multicêntrica", exemplificada pelos casos da Alemanha e dos Estados Unidos, nos quais a marca de 50\% do pessoal empregado em P\&D é obtida apenas após a soma de pelo menos oito regiões diferentes. No caso da Alemanha, as duas regiões que mais concentram pesquisadores são Munique e Stuttgard (cada uma com 12\% do total). No caso dos Estados Unidos, New Jersey tem 9\% e Boston tem 8\% dos pesquisadores.

Para comparação com essas informações, a tabela 1 apresenta os dados para o Brasil da distribuição de pesquisadores cadastrados no CNPq. Uma diferença com relação aos dados apresentados pelo BMBF (1999) é a nãoinclusão de pesquisadores atuando no setor produtivo na base do CNPq (que envolve fundamentalmente pesquisadores da área acadêmica e de algumas empresas estatais). As nove regiões metropolitanas mais as microrregiões de Campinas e São José dos Campos, apresentadas na tabela 1, concentram mais de $58 \%$ dos pesquisadores cadastrados no CNPq envolvidos em pesquisas nas áreas das ciências exatas e naturais. ${ }^{10}$ 
Tabela 1: Total de pesquisadores por regiões metropolitanas brasileiras (2000)

\begin{tabular}{lccc}
\hline Região metropolitana & Pesquisadores & \% da região metropolitana & \% do Brasil \\
\hline São Paulo (incluindo Campinas) & 9.539 & 33,5 & 19,51 \\
\hline Rio de Janeiro & 6.766 & 23,77 & 13,83 \\
\hline Porto Alegre & 2.572 & 9,03 & 5,26 \\
\hline Belo Horizonte & 2.306 & 8,1 & 4,72 \\
\hline Recife & 2.170 & 7,62 & 4,44 \\
\hline Curitiba & 1.283 & 4,51 & 2,62 \\
\hline São José dos Campos & 1.139 & 4 & 2,33 \\
\hline Fortaleza & 1.054 & 3,7 & 2,16 \\
\hline Salvador & 892 & 3,13 & 1,82 \\
\hline Belém & 748 & 2,63 & 1,53 \\
\hline Total das regiões metropolitanas & 28.469 & 100 & 58,21 \\
\hline Total do país & 48.906 & - & - \\
\hline
\end{tabular}

Fonte: CNPq, 2000 (elaboração própria).

Para comparação com os dados internacionais, nessa tabela estão somados os dados de São Paulo e Campinas, porque essas regiões têm uma forte articulação espacial similar à existente nas regiões que compõem a Grande Paris e a Grande Londres, conforme os dados compilados pelo BMBF (1999). A tabela 1 mostra que do total de pesquisadores das RMs, São Paulo (incluindo a microrregião de Campinas) e Rio de Janeiro ocupam o topo da lista, com 19,51\% e 13,83\% da produção nacional, respectivamente. Em seguida, surgem Porto Alegre e Belo Horizonte com 5,26\% e 4,72\% do total do país. O Brasil tem uma distribuição de pessoal em P\&D mais concentrada do que a da Alemanha. Os EUA, por sua vez, têm uma estrutura mais bem distribuída, mais multicêntrica.

Comparando o padrão de distribuição de pesquisadores com a distribuição geográfica dos resultados das atividades de P\&D (patentes e artigos científicos), são encontrados resultados diferentes - maior concentração. A tabela 2 apresenta a distribuição de acordo com as patentes depositadas entre 1990 e 2000.

A distribuição das patentes depositadas no INPI entre 1990 e 2000 pelas regiões de interesse demonstram que a produção tecnológica é mais concentrada do que a distribuição de pesquisadores. Neste caso, a região de São Paulo tem 39\% das patentes do país. Rio de Janeiro vem em segundo lugar, com pouco mais de $10 \%$ do total nacional. Neste ponto, nota-se que, em termos da distribuição das patentes, as regiões constituiriam quatro grupos, 
Tabela 2: Total de patentes depositadas no INPI por regiões metropolitanas e microrregiões selecionadas entre 1990 e 2000

\begin{tabular}{lccc}
\hline Região & Patentes & \% das regiões & \% do Brasil \\
\hline São Paulo & 3219 & 48,28 & 39,06 \\
\hline Interior de SP & 890 & 13,35 & 10,80 \\
\hline Rio de Janeiro & 843 & 12,64 & 10,23 \\
\hline Porto Alegre & 443 & 6,64 & 5,38 \\
\hline Campinas & 403 & 6,04 & 4,89 \\
\hline Belo Horizonte & 368 & 5,52 & 4,47 \\
\hline Curitiba & 322 & 4,83 & 3,91 \\
\hline São José dos Campos & 74 & 1,11 & 0,90 \\
\hline Salvador & 63 & 0,94 & 0,76 \\
\hline Recife & 22 & 0,33 & 0,27 \\
\hline Fortaleza & 18 & 0,27 & 0,22 \\
\hline Belém & 3 & 0,04 & 0,04 \\
\hline Subtotal & 6.668 & 100 & 80,91 \\
\hline Resto do Brasil & 1.573 & - & 19,09 \\
\hline Total & 8.241 & - & 100
\end{tabular}

Fonte: INPI, 2002 (elaboração própria).

um a mais que no caso de pesquisadores. Assim, no extremo superior, estaria São Paulo; em seguida, bem mais abaixo, estaria o Rio de Janeiro, estas duas regiões se diferenciando significativamente das demais. As sete regiões restantes poderiam ser classificadas em outros dois grupos, sendo que Porto Alegre (5,38\%), Campinas (4,89\%), Belo Horizonte (4,47\%) e Curitiba $(3,91 \%)$ estariam em um grupo, enquanto São José dos Campos $(0,908 \%)$, Salvador $(0,76 \%)$, Recife $(0,27 \%)$, Fortaleza $(0,22 \%)$ e Belém $(0,04 \%)$ comporiam o grupo mais baixo. Outro aspecto importante a perceber na tabela 2 é a maior concentração das patentes em comparação com a concentração de pesquisadores, tanto dentro das regiões de interesse, como visto acima, quanto no Brasil, dado que as nove regiões concentram cerca de $70 \%$ da produção tecnológica do país (somado ao "interior paulista”, esse número alcança $81 \%$ do total brasileiro).

A distribuição da produção científica está apresentada na tabela 3.

A tabela 3 indica que no conjunto, a concentração da produção científica é similar à tecnológica. Mas, no interior desse conjunto, a distribuição da produção científica é menos assimétrica do que a tecnológica: São Paulo detém 19,19\% da produção científica, contra os 39,09\% da produção tecnológica. 
Tabela 3: Artigos científicos indexados no ISI com a participação brasileira em 1999 por regiões metropolitanas e microrregiões geográficas selecionadas

\begin{tabular}{lccc}
\hline Região & Artigos & \% das regiões & \% do Brasil \\
\hline São Paulo & 2.060 & 23,65 & 19,19 \\
\hline Rio de Janeiro & 1.759 & 20,19 & 16,39 \\
\hline Interior de SP & 1.537 & 17,64 & 14,32 \\
\hline Campinas & 1.044 & 11,98 & 9,73 \\
\hline Belo Horizonte & 664 & 7,62 & 6,19 \\
\hline Porto Alegre & 547 & 6,28 & 5,10 \\
\hline Curitiba & 268 & 3,08 & 2,50 \\
\hline Recife & 237 & 2,72 & 2,21 \\
\hline São José dos Campos & 173 & 1,99 & 1,61 \\
\hline Fortaleza & 162 & 1,86 & 1,51 \\
\hline Salvador & 143 & 1,64 & 1,33 \\
\hline Belém & 117 & 1,34 & 1,09 \\
\hline Subtotal & 8.711 & 100 & 81,16 \\
\hline Resto do Brasil & 2.022 & - & 18,84 \\
\hline Total & 10.733 & - & 100 \\
\hline
\end{tabular}

Fonte: ISI, 2000; RAIS, 1997 (elaboração própria).

Os dados apresentados nesta seção permitem algumas considerações de caráter introdutório. Em primeiro lugar, se o número de pesquisadores puder ser tomado como uma referência para o potencial para pesquisa e desenvolvimento, e, logo, para a produção científica e tecnológica, vê-se que as regiões de interesse dividem de forma mais próxima, em relação aos demais indicadores, esse potencial com o restante do país. Mais especificamente, as regiões do Rio de Janeiro e São Paulo não se descolam tanto das demais regiões vis-à-vis a produção de patentes e artigos.

Em segundo lugar, olhando para a distribuição das patentes, percebe-se não só que as regiões metropolitanas de São Paulo e Rio de Janeiro dominam maciçamente a produção tecnológica do país, como também que a região de São Paulo é, sem dúvida, a maior liderança neste campo. Este predomínio, primeiro das regiões metropolitanas, depois da região de São Paulo, na produção tecnológica está, de certo, relacionado: (1) ao volume de renda que circula nestas regiões; (2) à composição setorial da economia, com maior peso das atividades industriais em comparação às outras regiões; (3) à composição da indústria com maior incidência de setores com propensão a patentear mais alta; (4) à presença maior de externalidades positivas, como a própria concentração de pesquisadores e artigos. 
Em terceiro lugar, o indicador de produção científica, artigos indexados, tem a especificidade de apresentar co-autorias, o que leva um artigo a ser contado para cada região em que possui um autor, representando assim que a atividade cientifica foi ali realizada. Isso leva a considerar (1) que a distribuição mais uniforme da produção científica se deve, em parte, a essa especificidade; (2) que, apesar desta melhor distribuição, São Paulo ainda possui uma importância relativa alta, sugerindo uma maior capacidade de gerar spillovers científicos para o restante do país, ao mesmo tempo em que se beneficia da capacidade das demais regiões; (3) que o tamanho da economia é fator importante para explicar a produção científica das regiões, uma vez que pode significar uma maior disponibilidade de recursos para a condução de pesquisas; (4) que o número e o tamanho das instituições de pesquisa são relevantes para explicar a produção de artigos, bem como a conectividade com outros centros, no Brasil e no exterior.

Em quarto lugar, uma comparação entre os dados das tabelas 3.2 e 3.3 sugere um ponto importante para avaliação: apenas três regiões têm sua participação relativa em termos de produção tecnológica superior à participação em termos da produção científica: São Paulo, Porto Alegre e Curitiba. Características do processo de industrialização do país podem explicar esses casos. Possivelmente perfis científicos de regiões parecem ser mais flexíveis e plásticos vis-à-vis padrões industriais e tecnológicos. De qualquer forma, a identificação de regiões com uma maior participação relativa da produção científica pode ser uma indicação de potenciais para a ampliação da produção tecnológica nessas regiões metropolitanas.

\section{ESPECIALIZAÇÕES TECNOLÓGICAS E CIENTÍFICAS DAS RMS}

A conjectura básica desta seção é a existência de diferentes áreas de especialização das diversas regiões. A tabela 4 apresenta as três classes CNAE (classificação do IBGE) mais freqüentes. As patentes acompanham de forma geral a concentração das atividades econômicas (refletindo a classificação industrial das empresas mais importantes da região). São Paulo tem a liderança da classe CNAE "fabricação de matérias plásticas", o "interior de São Paulo" concentra-se na classe "fabricação de máquinas e equipamentos para agricultura, avicultura", o Rio de Janeiro, refletindo o peso da Petrobras, tem a liderança de "extração de petróleo", Belo Horizonte tem a liderança da 
Tabela 4: Patentes depositadas no INPI com primeiro titular nas regiões e microrregiões selecionadas segundo as três classes CNAE mais freqüentes

\begin{tabular}{|c|c|c|c|c|c|c|c|}
\hline \multirow{2}{*}{$\frac{\text { Região }}{\text { São Paulo }}$} & \multirow{3}{*}{$\begin{array}{l}\text { Classe CNAE } \\
\text { Fabricação de artefatos diversos } \\
\text { de plástico }\end{array}$} & \multirow{2}{*}{$\frac{\text { Patentes }}{211}$} & \multirow{3}{*}{$\frac{\text { Estado }}{4.617}$} & \multicolumn{4}{|c|}{ Região \% Estado\% Região IES } \\
\hline & & & & 3.219 & 4,57 & 6,55 & 0,99 \\
\hline & & & & & & & \\
\hline & Fabricação de outros aparelhos & 188 & 4.617 & 3.219 & 4,07 & 5,84 & 2,35 \\
\hline & eletrodomésticos & & & & & & \\
\hline & Sedes de empresas e unidades & 152 & 4.617 & 3.219 & 3,29 & 4,72 & 2,21 \\
\hline & administrativas locais & & & & & & \\
\hline \multirow[t]{4}{*}{ Interior de SP } & Fabricação de máquinas e equipamentos & 161 & 4.617 & 890 & 3,49 & 18,09 & 5,18 \\
\hline & para agricultura, avicultura & & & & & & \\
\hline & Fabricação de artefatos diversos de plástico & 067 & 4.617 & 890 & 1,45 & 7,53 & 1,14 \\
\hline & Produção de laminados planos de aço & 38 & 4.617 & 890 & 0,82 & 4,27 & 1,62 \\
\hline \multirow[t]{3}{*}{ Rio de Janeiro } & Extração de petróleo e gás natural & 184 & 880 & 843 & 20,91 & 21,83 & 9,78 \\
\hline & Produção de laminados planos de aço & 120 & 880 & 843 & 13,64 & 14,23 & 5,41 \\
\hline & Extração de minério de metais preciosos & 98 & 880 & 843 & 11,14 & 11,63 & 9,78 \\
\hline \multirow[t]{4}{*}{ Porto Alegre } & Fabricação de aparelhos de ar-condicionado & do 34 & 927 & 443 & 3,67 & 7,67 & 17,57 \\
\hline & Fabricação de máquinas e equipamentos & 32 & 927 & 443 & 3,45 & 7,22 & 15,67 \\
\hline & para as indústrias do vestiário & & & & & & \\
\hline & Fabricação de escovas, pincéis e vassouras & 30 & 927 & 443 & 3,24 & 6,77 & 8,33 \\
\hline \multirow[t]{4}{*}{ Campinas } & Educação superior & 108 & 4.617 & 403 & 2,34 & 26,8 & 9,48 \\
\hline & Fabricação de artefatos diversos de plástico & 0 57 & 4.617 & 403 & 1,23 & 14,14 & 2,15 \\
\hline & Fabricação de fogões, refrigeradores & 29 & 4.617 & 403 & 0,63 & 7,2 & 3,03 \\
\hline & e máquinas de lavar e secar & & & & & & \\
\hline \multirow[t]{4}{*}{ Belo Horizonte } & Produção de laminados não planos de aço & 48 & 611 & 368 & 7,86 & 13,04 & 14,72 \\
\hline & Educação Superior & 41 & 611 & 368 & 6,71 & 11,14 & 3,94 \\
\hline & Comércio varejista de outros produtos & 18 & 611 & 368 & 2,95 & 4,89 & 4,69 \\
\hline & não especificados anteriormente & & & & & & \\
\hline
\end{tabular}

“produção de laminados”, São José dos Campos “defesa” etc. Enfim, há uma nítida diferenciação entre as diversas regiões.

Para os objetivos propostos, é interessante notar na tabela 4 a posição da classe "educação superior" em Campinas e em Belo Horizonte. Essa classe está na liderança das duas RMs em função de suas universidades (Unicamp e URMG). A presente leitura sugere que esse dado indica um potencial para o setor produtivo. A posição de liderança de universidades em termos de patenteamento não parece ser elemento positivo, pois indica um problema na divisão de trabalho implícita no conceito de sistema de inovação. Uma evidência empírica disso pode ser encontrada em uma comparação simples: enquanto na lista dos 20 maiores patenteadores dos Estados Unidos há apenas uma universidade (Universidade da Califórnia, na $14^{\mathrm{a}}$ posição), no caso brasileiro há cinco universidades/instituições de pesquisa entre os 20 maio- 
Tabela 4 (cont.)

\begin{tabular}{|c|c|c|c|c|c|c|c|}
\hline \multirow{2}{*}{$\begin{array}{l}\text { Região } \\
\text { Curitiba }\end{array}$} & \multirow{3}{*}{$\begin{array}{l}\text { Classe CNAE } \\
\text { Fabricação de fogões, refrigeradores } \\
\text { e máquinas de lavar e secar }\end{array}$} & \multicolumn{2}{|c|}{ Patentes Estado } & \multicolumn{4}{|c|}{ Região \% Estado\% Região IES } \\
\hline & & 56 & 426 & 322 & 13,15 & 17,39 & 7,31 \\
\hline & & & & & & & \\
\hline & Fabricação de fios, cabos e condutores & 17 & 426 & 322 & 3,99 & 5,28 & 12,8 \\
\hline & elétricos isolados & & & & & & \\
\hline & $\begin{array}{l}\text { Fabricação de aparelhos receptores de } \\
\text { rádio e televisão e de reprodução }\end{array}$ & 15 & 426 & 322 & 3,52 & 4,66 & 6,86 \\
\hline São José & Defesa & 24 & 4617 & 74 & 0,52 & 32,43 & 106,91 \\
\hline \multirow[t]{3}{*}{ dos Campos } & Fabricação de peças e acessórios de metal & 7 & 4617 & 74 & 0,15 & 9,46 & 4,9 \\
\hline & para veículos automotores & & & & & & \\
\hline & $\begin{array}{l}\text { Fabricação de aparelhos telefônicos, } \\
\text { sistemas de intercomunicação }\end{array}$ & 6 & 4617 & 74 & 0,13 & 8,11 & 17,58 \\
\hline \multirow[t]{3}{*}{ Salvador } & Administração pública em geral & 31 & 64 & 63 & 48,44 & 49,21 & 32,7 \\
\hline & Fabricação de artefatos diversos de plástico & o 14 & 64 & 63 & 21,88 & 22,22 & 3,37 \\
\hline & Obras de urbanização e paisagismo & 3 & 64 & 63 & 4,69 & 4,76 & 130,81 \\
\hline \multirow[t]{3}{*}{ Recife } & Fabricação de gases industriais & 7 & 22 & 22 & 31,82 & 31,82 & 374,59 \\
\hline & Administração pública em geral & 3 & 22 & 22 & 13,64 & 13,64 & 9,06 \\
\hline & $\begin{array}{l}\text { Fabricação de artefatos de concreto, } \\
\text { cimento, fibrocimento, gesso }\end{array}$ & 2 & 22 & 22 & 9,09 & 9,09 & 21,41 \\
\hline \multirow[t]{4}{*}{ Fortaleza } & $\begin{array}{l}\text { Fabricação de outros aparelhos ou } \\
\text { equipamentos elétricos }\end{array}$ & 7 & 30 & 18 & 23,33 & 38,89 & 33,04 \\
\hline & Fabricação de material eletrônico básico & 4 & 30 & 18 & 13,33 & 22,22 & 29,07 \\
\hline & Fabricação de material elétrico para & 3 & 30 & 18 & 10 & 16,67 & 41,62 \\
\hline & instalações em circuito de consumo & & & & & & \\
\hline \multirow[t]{2}{*}{ Belém } & Outras atividades de serviços prestados & 3 & 3 & 3 & 100 & 100 & 84,09 \\
\hline & principalmente às empresas & & & & & & \\
\hline
\end{tabular}

Fonte: INPI, 2000 (elaboração própria).

res patenteadores (Albuquerque, 2003). Em termos de regiões brasileiras, o mesmo não ocorre com São Paulo, apesar da presença da USP. Ou seja, a posição de destaque de uma universidade pode ser indício de timidez do setor produtivo (e da necessidade de medidas para ampliar o envolvimento do setor produtivo nas atividades inovativas).

A tabela 5 organiza os dados de acordo com as classes tecnológicas das patentes. Nota-se uma razoável diversidade tecnológica das regiões estudadas. São Paulo tem especializações próximas às do conjunto do país (refletindo o peso da região sobre o conjunto do país), situando-se em "transporte, embalagem" e "móveis e aparelhos domésticos". O "interior paulista" tem a liderança de "agricultura", Rio de Janeiro "perfuração de solo", Porto Alegre "calçados", Belo Horizonte "medição e verificação" seguida de "química orgânica". Na tabela 5, agora focando a classe das patentes, vê-se para 
Tabela 5: Patentes indexadas no INPI com a participação brasileira de 1990 a 2000 por regiões metropolitanas e microrregiões geográficas selecionadas segundo as $\mathbf{3}$ classes mais freqüentes

\begin{tabular}{|c|c|c|c|c|c|c|c|}
\hline \multirow{4}{*}{$\begin{array}{l}\text { Região } \\
\text { São Paulo }\end{array}$} & \multirow{2}{*}{$\begin{array}{l}\text { Classe WIPO } \\
\text { Transporte, embalagem, } \\
\text { armazenamento, manipulação } \\
\text { de material delgado ou filamentar }\end{array}$} & \multirow{2}{*}{$\frac{\text { Patentes }}{423}$} & \multirow{2}{*}{$\begin{array}{c}\text { Estado } \\
4.617\end{array}$} & \multirow{2}{*}{$\frac{\text { Região }}{3.219}$} & \multicolumn{2}{|c|}{ \% Estado \% Região } & \multirow{2}{*}{$\frac{\mathrm{IET}}{1,37}$} \\
\hline & & & & & 9,16 & 13,14 & \\
\hline & $\begin{array}{l}\text { Móveis, artigos ou aparelhos } \\
\text { domésticos, moinhos de café, } \\
\text { moinhos de especiarias, } \\
\text { aspiradores em geral }\end{array}$ & 351 & 4.617 & 3.219 & 7,6 & 10,9 & 1,18 \\
\hline & $\begin{array}{l}\text { Ciência médica ou veterinária, } \\
\text { higiene }\end{array}$ & 229 & 4.617 & 3.219 & 4,96 & 7,11 & 1,41 \\
\hline \multirow[t]{3}{*}{ Interior de SP } & $\begin{array}{l}\text { Agricultura, silvicultura, pecuária, } \\
\text { caça, captura em armadilhas }\end{array}$ & 132 & 4.617 & 890 & 2,86 & 14,83 & 3,58 \\
\hline & $\begin{array}{l}\text { Transporte, embalagem, } \\
\text { armazenamento, manipulação de } \\
\text { material delgado ou filamentar }\end{array}$ & 73 & 4.617 & 890 & 1,58 & 8,2 & 0,85 \\
\hline & $\begin{array}{l}\text { Móveis, artigos ou aparelhos } \\
\text { domésticos, moinhos de café, } \\
\text { moinhos de especiarias, aspiradores } \\
\text { em geral }\end{array}$ & 53 & 4.617 & 890 & 1,15 & 5,96 & 0,65 \\
\hline \multirow[t]{3}{*}{ Rio de Janeiro } & Perfuração de solo, mineração & 76 & 880 & 843 & 8,64 & 9,02 & 8,08 \\
\hline & Medição, verificação & 67 & 880 & 843 & 7,61 & 7,95 & 2,47 \\
\hline & $\begin{array}{l}\text { Transporte, embalagem, } \\
\text { armazenamento, manipulação de } \\
\text { material delgado ou filamentar }\end{array}$ & 53 & 880 & 843 & 6,02 & 6,29 & 0,65 \\
\hline \multirow[t]{3}{*}{ Porto Alegre } & Calçados & 65 & 927 & 443 & 7,01 & 14,67 & 8,96 \\
\hline & $\begin{array}{l}\text { Móveis, artigos ou aparelhos } \\
\text { domésticos, moinhos de café, } \\
\text { moinhos de especiarias, } \\
\text { aspiradores em geral }\end{array}$ & 42 & 927 & 443 & 4,53 & 9,48 & 1,03 \\
\hline & $\begin{array}{l}\text { Transporte, embalagem, } \\
\text { armazenamento, manipulação de } \\
\text { material delgado ou filamentar }\end{array}$ & 38 & 927 & 443 & 4,1 & 8,58 & 0,89 \\
\hline \multirow[t]{4}{*}{ Campinas } & $\begin{array}{l}\text { Móveis, artigos ou aparelhos } \\
\text { domésticos, moinhos de café, } \\
\text { moinhos de especiarias, } \\
\text { aspiradores em geral }\end{array}$ & 39 & 4.617 & 403 & 0,84 & 9,68 & 1,05 \\
\hline & $\begin{array}{l}\text { Ciência médica ou veterinária, } \\
\text { higiene }\end{array}$ & 29 & 4.617 & 403 & 0,63 & 7,2 & 1,43 \\
\hline & $\begin{array}{l}\text { Transporte, embalagem, } \\
\text { armazenamento, manipulação de } \\
\text { material delgado ou filamentar }\end{array}$ & 29 & 4.617 & 403 & 0,63 & 7,2 & 0,75 \\
\hline & $\begin{array}{l}\text { Elementos ou unidades de } \\
\text { engenharia, medidas gerais } \\
\text { para assegurar e manter o bom } \\
\text { funcionamento efetivo de máquina } \\
\text { ou instalações, isolamento térmico } \\
\text { em geral }\end{array}$ & $\begin{array}{l}22 \\
\text { las } \\
0\end{array}$ & 4.617 & 403 & 0,48 & 5,46 & 1,26 \\
\hline \multirow[t]{3}{*}{ Belo Horizonte } & Medição, verificação & 30 & 611 & 368 & 4,91 & 8,15 & 2,54 \\
\hline & Química orgânica & 26 & 611 & 368 & 4,26 & 7,07 & 5,39 \\
\hline & Edificação & 23 & 611 & 368 & 3,76 & 6,25 & 2,16 \\
\hline
\end{tabular}


Tabela 5 (cont.)

\begin{tabular}{|c|c|c|c|c|c|c|c|}
\hline \multirow[t]{3}{*}{ Curitiba } & $\begin{array}{l}\text { Móveis, artigos ou aparelhos } \\
\text { domésticos, moinhos de café, } \\
\text { moinhos de especiarias, } \\
\text { aspiradores em geral }\end{array}$ & 44 & 426 & 322 & 10,33 & 13,66 & 1,48 \\
\hline & $\begin{array}{l}\text { Refrigeração ou resfriamento, } \\
\text { fabricação ou armazenamento } \\
\text { de gelo, liquefação ou } \\
\text { solidificação dos gases }\end{array}$ & 37 & 426 & 322 & 8,69 & 11,49 & 10,29 \\
\hline & $\begin{array}{l}\text { Transporte, embalagem, } \\
\text { armazenamento, manipulação } \\
\text { de material delgado ou filamentar }\end{array}$ & 35 & 426 & 322 & 8,22 & 10,87 & 1,13 \\
\hline \multirow{4}{*}{$\begin{array}{l}\text { São José } \\
\text { dos Campos }\end{array}$} & Elementos elétricos básicos & 10 & 4.617 & 74 & 0,22 & 13,51 & 4,86 \\
\hline & Medição, verificação & 7 & 4.617 & 74 & 0,15 & 9,46 & 2,94 \\
\hline & $\begin{array}{l}\text { Elementos ou unidades de } \\
\text { engenharia, medidas gerais para } \\
\text { assegurar e manter o bom } \\
\text { funcionamento efetivo das } \\
\text { máquinas ou instalações, } \\
\text { isolamento térmico em geral }\end{array}$ & 7 & 4.617 & 74 & 0,15 & 9,46 & 2,18 \\
\hline & $\begin{array}{l}\text { Compostos macromolecular } \\
\text { orgânicos, sua preparação ou seu } \\
\text { processamento químico, composiçõe } \\
\text { baseadas nos mesmos }\end{array}$ & $\begin{array}{l}5 \\
\text { es }\end{array}$ & 4.617 & 74 & 0,11 & 6,76 & 6,19 \\
\hline \multirow[t]{3}{*}{ Salvador } & Cômuto, cálculo, contagem & 9 & 64 & 63 & 14,06 & 14,29 & 11,21 \\
\hline & Técnica de comunicação elétrica & 6 & 64 & 63 & 9,38 & 9,52 & 5,45 \\
\hline & $\begin{array}{l}\text { Móveis, artigos ou aparelhos } \\
\text { domésticos, moinhos de café, } \\
\text { moinhos de especiarias, } \\
\text { aspiradores em geral }\end{array}$ & 5 & 64 & 63 & 7,81 & 7,94 & 0,86 \\
\hline \multirow[t]{5}{*}{ Recife } & $\begin{array}{l}\text { Tratamento de águas } \\
\text { residuais, de esgoto, ou de } \\
\text { lamas e lodos }\end{array}$ & 7 & 22 & 22 & 31,82 & 31,82 & 58,27 \\
\hline & Edificação & 2 & 22 & 22 & 9,09 & 9,09 & 3,13 \\
\hline & Medição, verificação & 2 & 22 & 22 & 9,09 & 9,09 & 2,83 \\
\hline & $\begin{array}{l}\text { Transporte, embalagem, } \\
\text { armazenamento, manipulação } \\
\text { de material delgado ou filamentar }\end{array}$ & 2 & 22 & 22 & 9,09 & 9,09 & 0,95 \\
\hline & $\begin{array}{l}\text { Ciência médica ou veterinária, } \\
\text { higiene }\end{array}$ & 1 & 22 & 22 & 4,55 & 4,55 & 0,9 \\
\hline \multirow[t]{4}{*}{ Fortaleza } & Sinalização & 5 & 30 & 18 & 16,67 & 27,78 & 38,15 \\
\hline & Iluminação & 2 & 30 & 18 & 6,67 & 11,11 & 18,69 \\
\hline & Dispositivos de verificação & 2 & 30 & 18 & 6,67 & 11,11 & 13,27 \\
\hline & $\begin{array}{l}\text { Educação, criptografia, } \\
\text { apresentação visual, anúncios } \\
\text { logotipos }\end{array}$ & 2 & 30 & 18 & 6,67 & 11,11 & 7,96 \\
\hline \multirow[t]{3}{*}{ Belém } & $\begin{array}{l}\text { Engenharia hidráulica, fundações, } \\
\text { terraplenagem }\end{array}$ & 1 & 3 & 3 & 33,33 & 33,33 & 124,86 \\
\hline & $\begin{array}{l}\text { Fundição, metalurgia de } \\
\text { pós-metálicos }\end{array}$ & 1 & 3 & 3 & 33,33 & 33,33 & 57,23 \\
\hline & $\begin{array}{l}\text { Máquinas, ferramentas, usinagem } \\
\text { de metal não incluída em outro local }\end{array}$ & 1 & 3 & 3 & 33,33 & 33,33 & 48,19 \\
\hline
\end{tabular}

Fonte: INPI, 2000 (elaboração própria). 
as microrregiões o mesmo retrato da tabela 2 para o Brasil: especializações em áreas tradicionais, basicamente relacionadas às engenharias. A tabela 5 também aponta as cidades possivelmente responsáveis pelo crescimento das áreas de saúde, biológica e química: São Paulo, Campinas e Belo Horizonte.

A tabela 6 apresenta os dados da produção científica e permite sugerir três diferentes "padrões de especialização", em termos das três discipinas líderes: (1) padrão "saúde”: Belo Horizonte, Curitiba e Belém têm todas as três disciplinas diretamente relacionadas à ciências da vida; (2) padrão "ciências exatas-engenharia": Campinas; (3) padrão "ciências da terra-exatas": São José dos Campos; (4) padrão "misto", que envolve regiões com predominância de exatas (Rio de Janeiro) e com predominância de saúde (São Paulo, Salvador, Recife e Porto Alegre).

$\mathrm{Na}$ tabela 6 nota-se ainda que a disciplina mais freqüente nas regiões metropolitanas avaliadas é "bioquímica e biologia molecular" (presente entre as disciplinas líderes em 5 das 11 regiões avaliadas). Quase todas as regiões apresentam entre as líderes disciplinas relacionadas à saúde em geral, com exceção de São José dos Campos e de Campinas.

Finalmente, o cotejamento entre as tabelas 5 e 6 indica a co-localização de atividades científicas e tecnológicas. Essa justaposição permite avaliar a existência de conexões ou desconexões entre as duas dimensões em nível local. O resultado inicial, decorrente da comparação entre as especializações científica e tecnológica, é a identificação de uma situação de generalizada "desconexão" entre as duas dimensões, com a possível exceção de São Paulo, onde a classe tecnológica "ciência médica ou veterinária" (WIPO) aparece entre as líderes e é facilmente relacionável com "bioquímica e biologia celular" e "cirurgia" entre as disciplinas científicas líderes. Dado o peso da área de saúde entre as RMs, uma avaliação particular sobre o setor saúde deve ser realizada (ver Vilela et al., 2004, para uma avaliação preliminar).

\section{CONCLUSÃO}

Este artigo indica a riqueza de informações que pode ser extraída de bases de dados de patentes, artigos e pesquisadores, quando a investigação toma como referência regiões metropolitanas. Entre as conclusões preliminares, seis pontos podem ser ressaltados quanto à distribuição das atividades científicas e tecnológicas do país: 
Tabela 6: Produção científica por região (1999)

\begin{tabular}{|c|c|c|c|c|c|c|c|}
\hline Região & Disciplina & Artigos & Estado & Região & $\%$ Estado & \%Região & IEC \\
\hline \multirow[t]{3}{*}{ São Paulo } & Bioquímica e biologia molecular & 134 & 7.998 & 3.350 & 1,68 & 4,00 & 1,01 \\
\hline & Ciências materiais, multidisciplinar & 109 & 7.998 & 3.350 & 1,36 & 3,25 & 1,00 \\
\hline & Cirurgia & 95 & 7.998 & 3.350 & 1,19 & 2,84 & 2,60 \\
\hline \multirow[t]{3}{*}{ Rio de Janeiro } & Física, multidisciplinar & 135 & 2.979 & 2.906 & 4,53 & 4,65 & 1,78 \\
\hline & Física da matéria condensada & 114 & 2.979 & 2.906 & 3,83 & 3,92 & 1,08 \\
\hline & Bioquímica e biologia molecular & 102 & 2.979 & 2.906 & 3,42 & 3,51 & 0,88 \\
\hline \multirow[t]{3}{*}{ Interior de SP } & Ciências materiais, multidisciplinar & 156 & 7.998 & 2.723 & 1,95 & 5,73 & 1,76 \\
\hline & Bioquímica e biologia molecular & 149 & 7.998 & 2.723 & 1,86 & 5,47 & 1,38 \\
\hline & Física da matéria condensada & 110 & 7.998 & 2.723 & 1,38 & 4,04 & 1,11 \\
\hline \multirow[t]{3}{*}{ Campinas } & Física da matéria condensada & 89 & 7.998 & 1.637 & 1,11 & 5,44 & 1,50 \\
\hline & Ciências materiais, multidisciplinar & 86 & 7.998 & 1.637 & 1,08 & 5,25 & 1,62 \\
\hline & Física aplicada & 67 & 7.998 & 1.637 & 0,84 & 4,09 & 2,05 \\
\hline \multirow[t]{3}{*}{ Belo Horizonte } & Ciência veterinária & 99 & 1.660 & 1.064 & 5,96 & 9,30 & 4,58 \\
\hline & Parasitologia & 52 & 1.660 & 1.064 & 3,13 & 4,89 & 2,60 \\
\hline & Medicina tropical & 47 & 1.660 & 1.064 & 2,83 & 4,42 & 2,33 \\
\hline \multirow[t]{4}{*}{ Porto Alegre } & Neurociências & 45 & 1.200 & 901 & 3,75 & 4,99 & 2,29 \\
\hline & Física da matéria condensada & 29 & 1.200 & 901 & 2,42 & 3,22 & 0,89 \\
\hline & Cirurgia & 27 & 1.200 & 901 & 2,25 & 3,00 & 2,75 \\
\hline & Ciências materiais, multidisciplinar & 27 & 1.200 & 901 & 2,25 & 3,00 & 0,92 \\
\hline \multirow[t]{3}{*}{ Curitiba } & Neurociências & 33 & 820 & 451 & 4,02 & 7,32 & 3,35 \\
\hline & Psquiatria & 29 & 820 & 451 & 3,54 & 6,43 & 5,20 \\
\hline & Bioquímica e biologia molecular & 20 & 820 & 451 & 2,44 & 4,43 & 1,12 \\
\hline \multirow[t]{3}{*}{ Recife } & Medicina tropical & 17 & 393 & 380 & 4,33 & 4,47 & 2,36 \\
\hline & Física da matéria condensada & 15 & 393 & 380 & 3,82 & 3,95 & 1,09 \\
\hline & Bioquímica e biologia molecular & 14 & 393 & 380 & 3,56 & 3,68 & 0,93 \\
\hline São José & Meteorologia e ciências & 25 & 7.998 & 288 & 0,31 & 8,68 & 19,08 \\
\hline \multirow[t]{3}{*}{ dos Campos } & Atmosféricas & & & & & & \\
\hline & Astronomia e astrofísica & 22 & 7.998 & 288 & 0,28 & 7,64 & 6,87 \\
\hline & Geociências, multidisciplinar & 17 & 7.998 & 288 & 0,21 & 5,90 & 7,73 \\
\hline \multirow[t]{3}{*}{ Fortaleza } & Física da matéria condensada & 25 & 267 & 254 & 9,36 & 9,84 & 2,71 \\
\hline & Farmacologia e farmácia & 21 & 267 & 254 & 7,87 & 8,27 & 3,56 \\
\hline & Agricultura multidisciplinar & 10 & 267 & 254 & 3,75 & 3,94 & 2,27 \\
\hline \multirow[t]{3}{*}{ Salvador } & Parasitologia & 17 & 264 & 221 & 6,44 & 7,69 & 4,09 \\
\hline & Medicina tropical & 16 & 264 & 221 & 6,06 & 7,24 & 3,83 \\
\hline & Química, multidisciplinar & 13 & 264 & 221 & 4,92 & 5,88 & 2,73 \\
\hline \multirow[t]{4}{*}{ Belém } & Parasitologia & 12 & 197 & 192 & 6,09 & 6,25 & 3,32 \\
\hline & Zoologia & 10 & 197 & 192 & 5,08 & 5,21 & 4,93 \\
\hline & Bioquímica e biologia molecular & 10 & 197 & 192 & 5,08 & 5,21 & 1,31 \\
\hline & Genética e hereditariedade & 9 & 197 & 192 & 4,57 & 4,69 & 2,92 \\
\hline
\end{tabular}

Fonte: ISI (1999). 
(1) as disparidades existente entre as participações relativas do Brasil na produção científica e na produção tecnológica mundiais justificam a busca de medidas e políticas que ampliem a capacidade do setor produtivo de aproveitar os conhecimentos gerados pela infra-estrutura científica do país;

(2) o padrão de distribuição das atividades de ciência e tecnologia no país difere de acordo com a variável avaliada, caracterizando-se por uma "concentração oligocêntrica moderada" em relação aos pesquisadores e à produção científica e por uma "concentração oligocêntrica forte" (quase alcançando uma "concentração monocêntrica") quando avalia-se a produção tecnológica;

(3) comparando a distribuição das atividades científicas e tecnológicas, apenas três regiões metropolitanas têm maior participação relativa em termos de tecnologia (São Paulo, Porto Alegre e Curitiba), o que levanta uma discussão em relação a um papel eventual de políticas locais viabilizadoras de um melhor aproveitamento das capacitações científicas disponíveis nessas regiões;

(4) a avaliação das especializações tecnológicas das regiões metropolitanas indica a existência de diversidade no país, na medida em que cada região tem em primeiro lugar um setor econômico diferente (classes CNAE) e uma classe tecnológica diferente (classificação WIPO) - apenas uma classe tecnológica repete-se em primeiro lugar em duas regiões diferentes: móveis e aparelhos domésticos em Curitiba e Campinas;

(5) as especializações científicas das regiões metropolitanas são também diversificadas, embora com mais similaridade (física da matéria condensada é líder em duas regiões, neurociência é líder em duas regiões e parasitologia é líder em duas regiões); disciplinas relacionadas à saúde estão entre as líderes de todas as regiões, com exceção de Campinas e São José dos Campos;

(6) quanto à "conexão" entre as dimensões científicas e tecnológicas em nível local, mais investigações são necessárias, mas é possível identificar um predomínio das situações de "desconexão" entre as duas dimensões. 


\section{NOTAS}

1. As subseções 1.1 e 1.2 apóiam-se em Albuquerque (2000).

2. O tema "sistemas nacionais de inovação" tem recebido crescente atenção na literatura. A partir das contribuições pioneiras de Freeman (1987) e de Nelson e Lundval na coletânea editada em Dosi et al. (1988), estudos, discussões e análises comparativas têm sido apresentados.

3. Para maiores detalhes sobre uma tipologia de sistemas de inovação, ver Albuquerque (1999). Sobre a correlação entre indicadores de produção científica, produção tecnológica e renda, ver Bernardes e Albuquerque (2003).

4. Petit (2005) apresenta uma atualização da literatura necessária (com forte ênfase nos países da OCDE) para dar conta dos impactos da emergência de uma economia baseada no conhecimento na elaboração de políticas públicas.

5. Rapini (2000) afirma que as séries temporais de artigos e patentes comportam-se de forma tal que a causalidade, no sentido de Granger, corre nos dois sentidos. Para mais detalhes, ver Rapini (2000).

6. Esse regime caracterizaria a situação de inúmeros países da África, que possuem alguma produção científica, mas nenhuma produção tecnológica identificada por patentes no USPTO (Banze, 2000).

7. Esse regime caracterizaria, por exemplo, o caso brasileiro (Rapini, 2000).

8. Os autores alertam para o problema de se considerar o "modelo linear invertido" que desconhece o papel da infra-estrutura científica na construção da "capacidade de absorção", indispensável para a imitação, para a adaptação de tecnologias disponíveis em países mais avançados e para a realização de inovações incrementais que caracterizam os movimentos iniciais nos processos de catching up. Ressaltam, portanto, a necessidade de combinar a crítica ao "modelo linear" com a crítica ao "modelo linear invertido", de modo a afirmar que há mútua determinação e feedbacks positivos entre as dimensões científica e tecnológica.

9. Para uma descrição detalhada dessa base de dados, ver Albuquerque (2003).

10. As regiões metropolitanas foram escolhidas de acordo com Diniz (2002). A inclusão das microrregiões de Campinas e São José dos Campos justifica-se porque são as duas únicas que aparecem tanto na lista das 20 regiões líderes em patentes depositadas como na lista das 20 líderes em produção científica.

\section{REFERÊNCIAS BIBLIOGRÁFICAS}

ALBUQUERQUE, E. (1999) "National systems of innovation and non-OECD countries: notes about a tentative typology". Revista de Economia Política, v. 19, n. 4, p. 35-52.

(2003) "Patentes e atividades inovativas: uma avaliação preliminar do caso brasileiro”. In: E. Viotti e M. M. Macedo (orgs.), Indicadores de ciência, tecnologia e inovação no Brasil. Campinas: Unicamp.

AMSDEN, A. H. (1989) Asia's next giant: South Korea and late industrialization. Nova York, Oxford: Oxford University Press. 
ANGEL, D. P. (1989) “The labor market for engineers in the US semi conductors industry". Economic Geography, 65(1), p. 99-112.

AUDRETSCH, D., FELDMAN, M. (1996) "R\&D spillovers and the geography of innovation and production”. American Economic Review, v. 86, n. 3, p. 630-640.

BANZE, C. E. (2000) A especificidade e a diversidade do continente africano: uma sugestão inicial de tipologias de sistemas nacionais de inovação. Monografia (Graduação em Ciências Econômicas), Universidade Federal de Minas Gerais, Belo Horizonte.

BERNARDES, A., ALBUQUERQUE, E. (2003) “Cross-over, thresholds and interactions between science and technology: lessons for less-developed countries”. Research Policy, v. 32, n. 5, p. $865-885$.

BMBF (1999) Zur technologischen Leistungsfähigkeit Deutschlands. Berlim: BMBF.

CAMAGNI, G. (1985) "Spatial diffusion of persuasive process innovation". Papers of the RSA, 58, p. 83-95.

CARLINO, G., CHATTERJEE, S., HUNT, R. (2001) Knowledge spillovers and the new economy of cities. Working Paper n. 01-14. Federal Reserve Bank of Philadelphia, Sept., p. 1-26.

CNPq (2000.) A pesquisa no Brasil. Disponível em <http://www.cnpq.br>

COHEN, W., LEVINTHAL, D. (1989) "Innovation and learning: the two faces of R\&D". The Economic Journal, v. 99, n. 397, p. 569-596.

— NELSON, R., WALSH, J. (2002) "Links and impacts: the influence of public R\&D on industrial research”. Management Science, v. 48, n. 1, p. 1-23.

DINIZ, C. C. (2002) “A nova configuração urbano-industrial no Brasil”. In: A. Kon, Unidade e fragmentação: a questão regional no Brasil. São Paulo: Perspectiva, p. 87-115.

DOSI, G., FREEMAN, C., NELSON, R., SILVERBERG, G., SOETE, L. (eds.) (1988) Technical change and economic theory. Londres: Pinter, p. 38-66.

FELDMAN, M., AUDRESTCH, D. (1999) Science-based diversity, specialization and localized competition. European Economic Review (43), p. 409-429.

FREEMAN, C. (1987) Technology policy and economic performance: lessons fron Japan. Londres: Pinter.

FRENKEL, A. (2001) "Why technology firms choose to locate in or near metropolitan areas". Urban Studies, 38(7), p. 1.083-1.101.

GOTTLIEB, P. D. (1995) "Residential amenities, firm location and economic development". Urban Studies, 32(9), p. 1.413-1.436.

INSTITUTE OF SCIENTIFIC INFORMATION (2000) (webofscience.fapesp.br).

JAFFE, A. B. (1989) “Real effects of academic research”. American Economic Review, v. 79, n. 5, p. 957-970.

— , TRAJTENBERG, M., HENDERSON, R. (1993) “Geographical localization of knowledge spillovers as evidenced by patents citations”. Quaterly Journal of Economics, v. 108, n. 3, p. 577-598.

JOHNSON, B., LUNDVALL, B. (2005) "Promovendo sistemas de inovação como resposta à economia do aprendizado crescentemente globalizada”. In: H. Lastres, J. Cassiolato, 
e A. Arroio, Conhecimento, sistemas de inovação e desenvolvimento. Rio de Janeiro: UFRJ/ Contraponto, p. 83-130.

KLEVORICK, A., LEVIN, R., NELSON, R., WINTER, S. (1995) “On the sources and significance of inter-industry differences in technological opportunities”. Research Policy, v. 24, p. $185-205$.

MALECKI, E. J. (1984) "High technology and local economic development". Journal of American Planning Association, 50(3), p. 262-269.

MARKUSSEN, A., HALL, P., GLASMEIER, A. (1986) High tech America: the what, how and why of sunrise industries. Boston, MA: Allen and Irwin.

NARIN, F., HAMILTON, K. S., OLIVASTRO, D. (1997) “The increasing linkage between U.S. technology and public science”. Research Policy, v. 26, n. 3, p. 317-330.

NELSON, R. (1996) Sources of economic growth. Cambridge, Mass.: Harvard University. (1982) “The role of knowledge in R\&D efficiency". The Quarterly Journal of Economics, Cambridge, v. 97, n. 3, p. 453-471.

(ed.) (1993) National innovation systems: a comparative analysis. Nova York, Oxford: Oxford University.

, ROSENBERG, N. (1993) “Technical innovation and national systems”. In: R. Nelson (ed.), National innovation systems: a comparative analysis. Nova York, Oxford: Oxford University, p. 3-21.

NSF (1996) Science \& engineering indicators 1996. Washington, DC: U.S. Government Printing Office. Disponível em < http://www.nsf.gov/sbe/srs/seind96/start.htm>.

PAVITT, K. (1991) "What makes basic research economically useful?". Research Policy, v. 20, n. 2, p. 109-119.

PEREZ, C., SOETE, L. (1988) "Catching up in technology: entry barriers and windows of opportunity”. In: G. Dosi, C. Freeman, R. Nelson et al. (eds.), Technical change and economic theory. Londres: Pinter, p. 458-479.

PETIT, P. (2005) "Estrututa e desenvolvimento de uma economia baseada no conhecimento: implicações para políticas públicas”. In: H. Lastres, J. Cassiolato e A. Arroio, Conhecimento, sistemas de inovação e desenvolvimento. Rio de Janeiro: UFRJ/Contraponto, p. 131-160.

RAPINI, M. S. (2000.) "Uma investigação sobre a relação de Granger-causalidade entre ciência e tecnologia para países em catching up e para o Brasil”. Monografia (Graduação em Ciências Econômicas), Universidade Federal de Minas Gerais, Belo Horizonte.

RELATÓRIO ANUAL DE INFORMAÇÕES SOCIAIS (1997). Ministério do Trabalho.

ROSENBERG, N. (1982) Inside the balck box: technology and economics. Cambridge: Cambridge University.

(1990) "Why do firms do basic research (with their money)?". Research Policy, v. 19, p. $165-174$.

SIVITANIDOU, R., SIVITANIDES, P. (1995) “The intrametropolitan distribution of R\&D activities: theory and empirical evidences". Journal of Regional Studies, 35(3), p. 391-415. 
USPTO (2001) (www.uspto.gov).

WADE, R. (1990) Governing the market: economy theory and the role of government in East Asian industrialization. Princeton: Princeton University Press.

WALLSTEN, S. J. (2001) "An empirical test of geographical knowledge spillovers using GIS and firm-level data”. Regional Science \& Urban Economics, 31(5), p. 571-599.

ZITT, M., RAMANANA-RAHARY, S., BASSECOUlARD, E., LAVILlE, F. (2003) "Potential science-technology spillovers in regions: an insight on geographic co-location of knowledge activities in the EU”. Scientometrics, v. 57, n. 2, p. 295-320. 